\section{Research Square}

Preprints are preliminary reports that have not undergone peer review.

They should not be considered conclusive, used to inform clinical practice, or referenced by the media as validated information.

\title{
Epidemiology of HIV-negative Rifampicin Resistance /Multidrug-Resistant Extrapulmonary Tuberculosis: 5 Years-experience From a National Tuberculosis Clinical Research Center in China
}

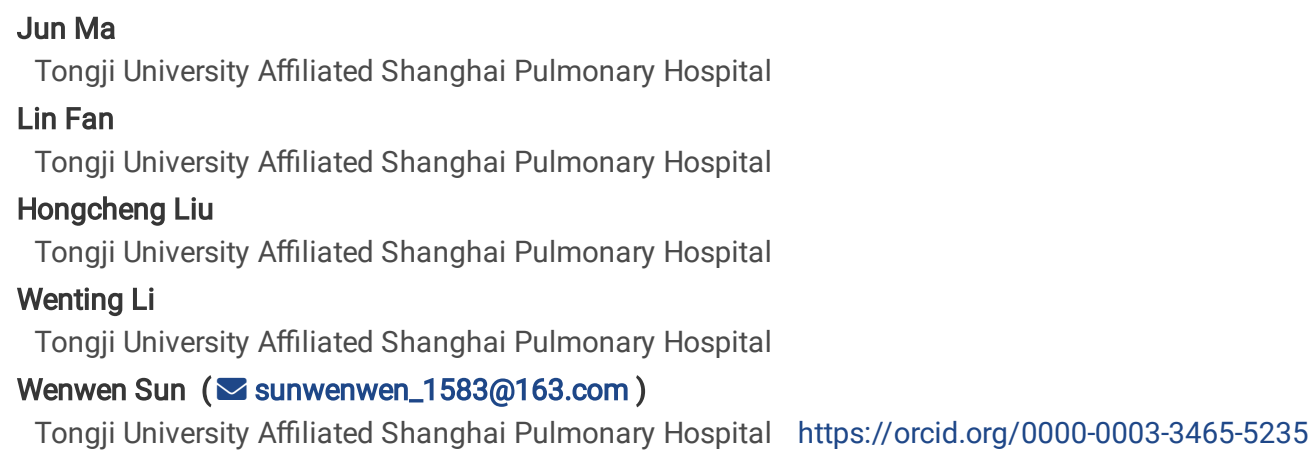

Tongji University Affiliated Shanghai Pulmonary Hospital

Lin Fan

Tongji University Affiliated Shanghai Pulmonary Hospital

Hongcheng Liu

Tongji University Affiliated Shanghai Pulmonary Hospital

Wenting Li

Tongji University Affiliated Shanghai Pulmonary Hospital

Wenwen Sun ( $\square$ sunwenwen_1583@163.com)

Tongji University Affiliated Shanghai Pulmonary Hospital https://orcid.org/0000-0003-3465-5235

\section{Research Article}

Keywords: China, Rifampicin Resistance, Multidrug Resistant, Extrapulmonary tuberculosis, Epidemiology, Xpert MTB/RIF

Posted Date: July 7th, 2021

DOI: https://doi.org/10.21203/rs.3.rs-636342/v1

License: (c) (i) This work is licensed under a Creative Commons Attribution 4.0 International License. Read Full License 


\section{Abstract}

Background: China is a region with a high global burden of Rifampicin Resistance 『Multidrug-Resistant tuberculosis (RR/MDR-TB) and low HIV incidence. Our aim was to assess the epidemiological and clinical characteristics of RR/MDR-extrapulmonary tuberculosis (EPTB) over the past five years in China to inform national TB control programmes.

Methods: We investigated the epidemiological and clinical datas of all MDR/RR-EPTB cases in a TB specialized hospitals in China over a five-year period and compared cases with a cohort of MDR/RR-PTB patients over the same period.

Results: Of the 1,700 RR/MDR-TB patients enrolled, $17.76 \%$ were EPTB. The incidence of RR囚MDR-EPTB were increasing.The most common anatomical site was pleura/chest wall (20.20\%). Compared with RR囚MDR-Pulmonary tuberculosis (PTBQRRR/MDR-EPTB were were predominately female $(50.66 \%, P<0.01) \bigotimes$ more prone to delay $(P<0.01)$ in RR/ MDR-EPTB detection with the median time of $145 \rrbracket 14,341 \rrbracket$ days since first visit $\rrbracket$ more less likely to develop diabetes $(22.85 \%, P<0.01) \rrbracket$ more likely to be newly diagnosed $(56.95 \%, P<0.01)$ هlower successful treatment outcome $869.56 \% \bigotimes$

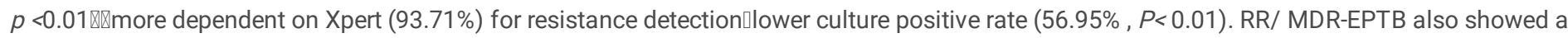
significantly higher rate of pre-XDR/XDR $(32.45 \%, P<0.01)$.

Conclusion: RR/MDR-EPTB is different from RR-MDR/PTB in both epidemiology and clinical. Clinicians should recommend Xpert in early stage and use fluoroquinolones cautiously in cases suspected TB.

\section{Introduction}

Of the 7.1 million newly diagnosed tuberculosis (TB) cases notified to World Health Organization (WHO) in 2019, 16\% were EPTB (1). EPTB can affect almost any organ and produces a wide range of clinical manifestations that pose challenges to effective diagnosis and management (2). Epidemiological studies recently indicate that the proportion of EPTB is steadily increasing all over the world including China $(3,4,5)$. According to the WHO, when PTB and EPTB are present together, EPTB is usually reported as PTB, and the diagnosis of EPTB is challenging, so the actual incidence of EPTB may be even higher(6).

Over the past 20 years, the incidence and death rates of TB have declined significantly as a result of global efforts to combat the disease. However, drug-resistant TB, especially Rifampicin resistant tuberculosis(RR-TB)and multidrug-resistant TB (MDR-TB), remain the major obstacle to ending the global TB epidemic (2). In 2019, about 465,000 cases of RR/TB occurred, of which 78\% were MDR-TB. In 2019, China accounted for $14 \%$ of the world's total RR/MDR-TB population, while the global MDR-TB treatment success rate was only 57\% (2). However, to our knowledge, epidemiological reports on RR/MDR-EPTB are still lacking. In recent years, with the wide range applications of Xpert MTB/RIFロXpert national regulatory concern on MDR-TB, MDR-EPTB has also increasingly attracted the attention of goverment and researchers. Considering the multiple organ-specific clinical symptoms and non-specific systemic symptoms, it is important to have a deeper understanding of the site distribution and epidemiological characteristics of RR/MDR-EPTB for the development of appropriate protocols to manage patients. However, the transmission mode and cause of RR/MDR-EPTB remain still unknown, and in-depth epidemiological investigation and relevant clinical information are also lacking. Therefore, we conducted a 5-year retrospective study to analyze the epidemiological and clinical characteristics of RR/MDR-EPTB under the new TB control model from a national tuberculosis clinical research center in China. To our knowledge, this is the first time that datas on RR/MDREPTB have been collected and discussed since the Xpert widely introduced in China from 2016.The results of the study may provide a theoretical basis for the control of the disease, especially in high TB burden areas.

\section{Materials And Methods}

\section{Ethics statement}

The retrospective study was conducted at a national TB clinical research center with nearly 300 beds that received the referrals for RR/MDR-TB patients from east China. In addition, the hospital is the only TB specialized hospital in Shanghai and the only national TB clinical center in eastern China. We undertakes the management of the regional RR/MDR-TB patients in cooperation with the Shanghai Center for Disease Control and Prevention (CDC). The retrospective study was approved by The Ethics Committee of Shanghai Pulmonary Hospital (Approval number k17-138 ).

\section{Data Sources and Collection}

We conducted a descriptive analysis of the demographic and clinical datas of RR/MDR-EPTB patients admitted to Shanghai Pulmonary Hospital from January 1, 2015 to December 31, 2019. The electronic medical record system recorded the patient's demographic characteristics and all clinical datas (including outpatient and inpatient records, examination and test reports, treatment and follow-up procedures,etc ). Datas during the study period were collected using Excel sheets.

\section{Definitions and Inclusion Criteria:}

The cases were classified according to the main anatomical site and reported as PTB or EPTB. The PTB group was defined as the lung as the only pathogenic anatomical site. EPTB group was defined as TB caused by mycobacterium tuberculosis (MTB) infecting any anatomical site other than

Page 2/10 
the lungs, with or without co-existent PTB $(7,8)$.

MDR-TB (at least resistant to isoniazid and rifampicin) Dextensively drug-resistant tuberculosis (XDR-TB, resistance to both fluoroquinolones and second-line injection agents) or pre-XDR (resistant to either fluoroquinolones or second-line injection agents) were defined through drug sensitivity test ( DST) based on MTB culture (9).

Resistance to Rifampicin is mainly associated with mutations in a limited region of the rpob gene (10), which may occur alone or may be associated with other drug resistance. In settings with a high burden of MDR-TB, the presence of resistance to rifampicin alone can serve as an alternative to MDR-TB (11). As recommended by the WHO for reporting purposes, we considered RR-TB as a proxy for MDR-TB, because rifampicin mono-resistant TB has been considered relatively rare (12). In the present study, resistance to rifampicin was determined based on MTB culture DST or genotype DST (Xpert). Shanghai CDC requires that all patients suspected TB should teste for Xpert when firstly diagnosed TB, as well as MTB smear and culture. In accordance with the recommendations of the Global Laboratory Initiative (GLI) and the World Health Organization (13), TB patients who are not at high risk of drug resistance should undergo two times of Xpert tests to determine the presence of drug resistance. In the present study, patients with newly diagnosed MDR/RR-EPTB with Xpert rifampicin resistant mutation results were given a second Xpert test to reduce the false positives.

Inclusion criteria: 1) MTB culture was positive, and MTB cultural-based DST indicated at least rifampicin resistance on extrapulmonary specimens. 2) Xpert MTB positive and rifampicin resistance detected on extrapulmonary specimens. 3) Complete clinical datas and regular follow-up.

The delay in TB detection is defined as the time (days) between the first visit to hospital and the date when RR/MDR-DST result is detected by DST or Xpert (14).

\section{Microbiological assessment}

Appropriate samples are taken from the anatomical site and sent to a microbiology laboratory. Body fluid /tissue samples were collected for smear microscopy and MTB culture. BACTECTM MGIT 960 culture (MGIT 960) was used to conduct the MTB culture and DST. BACTEC MGIT960 system, MGIT culture tube and SIRE kit were purchased from BD company from United States. According to the instructions of the Gene Xpert MTB/RIF (Xpert) testing system (Cepheid, USA), homogenate tissues were mixed with GeneXpert reagent, incubated at room temperature for a short period, and then transferred to the cartridge and inserted into GeneXpert device. The test results were automatically interpreted after 2 hours.

\section{Treatment regimens and follow-up:}

RR/MDR-EPTB patients received the diagnosis and protocol design from the expert team of the hospital and Shanghai CDC. Treatment regimens were individualized according to WHO guidelines and DST results (15). All enrolled patients were regularly treated and followed up, and direct observation therapy (DOT) was used throughout the treatment process.

The specialist evaluates the patient on a monthly basis. Monthly physical examination, complete blood count, erythrocyte sedimentation rate, liver and kidney function examination. Radiological examination or invasive surgery is performed when necessary. Treatment outcome datas (success, failed, died, still on treatment) were collected 18 months after start of treatment. Treatment outcome was considered successful if a case was cured or had completed treatment.

\section{Statistical analysis}

Intergroup differences (PTB and EPTB; Different anatomical site of EPTB) were determined by the chi-square test or Fisher's exact test to obtain significant factors. For each anatomical site, we also listed the proportion of cases by the year in which the patient sought treatment. Chi-square trend test was used to analyze the changing trend of the proportion of cases in different anatomical sites.Statistical associations were expressed as odds ratios (OR) to $95 \%$ confidence intervals $(\mathrm{Cl})$. If $P<0.01$, the difference was considered statistically significant. Statistical analysis was performed using Stata 14 software (Statacorp, Texas, United States (US)).

\section{Results}

\section{Study population and extrapulmonary anatomical site distribution:}

A total of 1743 RR/MDR-TB patients were recorded in the electronic case database of Shanghai pulmonary hospital from January 2015 to December 2019.12. After excluding 43 patients with incomplete clinical datas, 1700 patients were included in the final statistics. Among them,

82.24\% $\$ 1398 / 1700 \rrbracket$ were PTB and $17.76 \% \varangle 302 / 1700 \rrbracket$ were EPTB. The most common anatomical site was pleura/chest wall (20.20\%囚61/302).

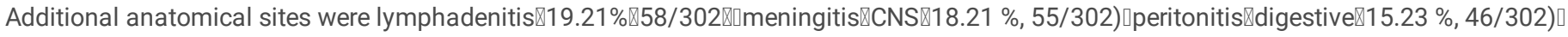

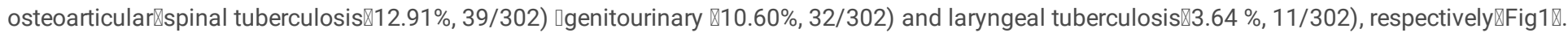

\section{Demographic analysis and clinical characteristics of RR/MDR-EPTB:}

The demographic and clinical characteristics of the patients are shown in Table 1. Compared with RR/MDR-PTB in the same period,the majority of EPTB patients were predominately female $(50.66 \% \square P<0.01) \rrbracket$ more prone to delay $(P<0.01)$ in RR/ MDR-EPTB detection with the median time of 
145 (14,341 days $\square$ more less likely to develop diabetes $(22.85 \%, P<0.01)$ and more likely to be newly diagnosed $(56.95 \%, P<0.01)$. In terms of microbiological characteristics, the culture positive rate of MDR/RR-EPTB was significantly lower (56.95\%, $P<0.01)$, and RR/MDR-EPTB also showed a significantly higher rate of pre-XDR/XDR $(32.45 \%, P<0.01)$.

Table 1

Comparison of clinical characteristics of RR/MDR-PTB Vs RR/MDR-EPTB during the same period, 2015-2019:

\begin{tabular}{|c|c|c|c|}
\hline Variable & RR/MDR-PTB $(n=1398)$ & RR/MDR-EPTB ( $n=302)$ & $P$ \\
\hline \multicolumn{4}{|l|}{$\operatorname{Sex}(n, \%)$} \\
\hline M & 995(71.39) & $149(49.33)$ & $*<0.01$ \\
\hline $\mathrm{F}$ & 403(28.83) & $153(50.66)$ & \\
\hline Median age, years (range) & $45(11,78)$ & $38(16,69)$ & 0.12 \\
\hline \multicolumn{4}{|l|}{ Residence (n,\%) } \\
\hline Rural & $895(64.02)$ & $180(59.60)$ & 1.58 \\
\hline Urban & $503(35.98)$ & 122(40.39) & \\
\hline \multicolumn{4}{|l|}{ TB History (n,\%) } \\
\hline newly & 418(29.90) & 172(56.95) & $*<0.01$ \\
\hline Retreated & $980(70.10)$ & $130(43.05)$ & \\
\hline Diabetes $(n, \%)$ & $579(41.42)$ & $69(22.85)$ & $*<0.01$ \\
\hline Median time to treatment initiation from first visit,Days (range) & $82(10,162)$ & $145(14,341)$ & $*<0.01$ \\
\hline Xpert positive (n,\%) & 1282(91.70) & $283(93.71)$ & 0.24 \\
\hline Culture positive (n,\%) & $985(70.46)$ & $172(56.95)$ & $*<0.01$ \\
\hline XDR /pre-XDR (n,\%) & 264(18.88) & $98(32.45)$ & $*<0.01$ \\
\hline Teatment success rate $(\mathrm{n}, \%)$ & $1105(79.21 \%)$ & $198(65.56)$ & $*<0.01$ \\
\hline
\end{tabular}

The median age was 38 (16-69) for RR/MDR-EPTB and $45(21-75)$ for RR/MDR-PTB (P0.12). The proportion of rural was 64.02 \% (895/1398) in RR/MDR-PTB and 59.60\% (180/302) in RR/MDR-PTB (P1.58). Xpert was positive in $91.70 \%(1282 / 1398)$ of RR/MDR-PTB and 93.71\% (283/302) of RR/MDR-EPTB. There were no significant differences between the above three items.

\section{Clinical characteristics of different anatomical sites of EPTB:}

In our study, women were more likely than men to have pleurisy खchest wall TB (OR 2.26, 95\% Cl 0.67-2.86) ㅁlymphadenitis TB (OR 1.64,95\% CI 1.49$1.81)$ and genitourinary TB (OR $1.28,95 \% \mathrm{Cl} 1.36-2.08)$; while men were more likely than women to develop peritonitis $₫$ digestive TB (OR $2.21,95 \% \mathrm{Cl}$ 1.67-4.86) 凶tuberculous meningitis/CNS (OR 1.76, 95\% Cl 0.67-2.86) पosteoarticular/spinal TB (OR 2.54, 95\% Cl 1.21-3.86) and laryngeal TB (OR 1.75, $95 \% \mathrm{Cl} 2.77-11.9)$. Pleural/chest wall TB tended to be relatively young with the median age of $19.8(24,65)$ years old while tuberculous meningitis/CNS tended to be relatively old with the median age of 48.1 (17,72》years old. Tuberculous meningitis/CNS (OR 3.32, 95\% Cl 1.35-2.73) $]$ Pleural/chest wall TB (OR 1.65, 95\% Cl 1.02-3.45) and osteoarticular》spinal TB (OR $2.93,95 \% \mathrm{Cl} 0.56-0.70)$ were more frequent in patients from rural than those from urban. In contrast, patients in urban were more likely to have peritonitis $₫$ digestive TB than those from rural (OR $1.33,95 \%$ CI 0.56 $0.78)$.

In terms of the TB history, lymphadenitis TB (OR 1.25, 95\% Cl 1.30-2.01) घpleural /chest wall TB (OR 1.61, 95\% Cl 1.65-2.82) $\square$ peritonitis凹digestive TB (OR 1.31, 95\% Cl 0.36-1.08) पtuberculous meningitis/CNS (OR 1.58, 95\% Cl 1.36-2.08) and genitourinary TB (OR 1.67, 95\% Cl 2.36-3.91) were mainly newly diagnosed.

The longer delay in RR/ MDR-TB detection was found in osteoarticular/spinal TB with the median time of 190 (60,341) days $\square$ genitourinary TB with the median time of 185 (30,320) days and pleural/chest wall TB with the median time of $151(21,271)$ days. (Table 2 ) 
Table 2

Clinical characteristics of RR/MDR-EPTB in different sites :

\begin{tabular}{|c|c|c|c|c|c|c|c|}
\hline Variable & $\begin{array}{l}\text { Lymphadenitis } \\
(n=58)\end{array}$ & $\begin{array}{l}\text { Pleurisy/chest } \\
\text { wall } \\
(n=61)\end{array}$ & $\begin{array}{l}\text { Peritonitis/digestive } \\
(n=46)\end{array}$ & $\begin{array}{l}\text { Meningitis/CNS } \\
(n=55)\end{array}$ & $\begin{array}{l}\text { Genitourinary } \\
(n=32)\end{array}$ & $\begin{array}{l}\text { Osteoarticular/spinal } \\
(n=39)\end{array}$ & $\begin{array}{l}\text { Laryngeal } \\
(n=11)\end{array}$ \\
\hline \multicolumn{8}{|l|}{$\operatorname{Sex}(n, \%)$} \\
\hline M & 24(41.38) & 20(32.79) & $31(67.39)$ & $35(63.64)$ & $14(43.75)$ & 28(71.79) & $7(63.64)$ \\
\hline $\mathrm{F}$ & $34(58.62)$ & $41(67.21)$ & 15(32.61) & $20(36.26)$ & $18(56.25)$ & $11(28.21)$ & $4(36.36)$ \\
\hline $\begin{array}{l}\text { Median } \\
\text { age, years } \\
\text { (range) }\end{array}$ & $24.6(15,58)$ & $19.8(24,65)$ & $45.4(32,67)$ & $48.1(17,72)$ & $47.7(29,70)$ & $40.3(19,68)$ & $28.6(16,61)$ \\
\hline \multicolumn{8}{|l|}{$\begin{array}{l}\text { Residence } \\
(n, \%)\end{array}$} \\
\hline Rural & $30(51.72)$ & $38(62.30)$ & $20(43.48)$ & $42(76.36)$ & 15(46.88) & 28(71.79) & $7(63.64)$ \\
\hline Urban & $28(48.28)$ & 23(37.70) & $26(56.52)$ & $13(23.64)$ & 17(53.13) & $11(28.21)$ & $4(36.36)$ \\
\hline \multicolumn{8}{|l|}{$\begin{array}{l}\text { TB } \\
\text { History } \\
(\mathrm{n}, \%)\end{array}$} \\
\hline newly & $32(55.17)$ & $35(57.38)$ & $26(56.52)$ & $35(63.64)$ & $20(62.50)$ & 19(48.72) & $5(45.45)$ \\
\hline Retreated & 26(44.83) & $26(42.62)$ & $20(43.48)$ & $20(36.36)$ & $12(37.50)$ & $20(51.28)$ & $6(54.55)$ \\
\hline $\begin{array}{l}\text { Diabetes } \\
(n, \%)\end{array}$ & 10(24.39) & $9(29.03)$ & $1(20.00)$ & $11(35.48)$ & $6(25.00)$ & $9(29.03)$ & $5(62.50)$ \\
\hline $\begin{array}{l}\text { Median } \\
\text { time to } \\
\text { treatment } \\
\text { initiation } \\
\text { from first } \\
\text { visit,Days } \\
\text { (range) }\end{array}$ & $117(18,169)$ & $151(21,271)$ & $128(14,160)$ & $41(18,262)$ & $185(30,320)$ & $190(60,341)$ & $32(14,121)$ \\
\hline $\begin{array}{l}\text { Xpert } \\
\text { positive } \\
(n, \%)\end{array}$ & 54 (93.10) & 57 (93.44) & $41(89.13)$ & $52(94.55)$ & $29(90.63)$ & 36 (92.31) & $\begin{array}{l}11 \\
(100.00)\end{array}$ \\
\hline $\begin{array}{l}\text { Culture } \\
\text { positive } \\
(n, \%)\end{array}$ & $33(56.90)$ & 39 (63.93) & $27(58.70)$ & $20(36.36)$ & 22 (68.75) & $21(53.85)$ & $10(90.91)$ \\
\hline $\begin{array}{l}\text { XDR /pre- } \\
\text { XDR (n,\%) }\end{array}$ & $13(22.41)$ & $15(15.31)$ & 12 (26.09) & 16 (29.09) & $16(50.00)$ & $24(61.54)$ & $2(18.18)$ \\
\hline $\begin{array}{l}\text { Teatment } \\
\text { success } \\
\text { rate }(n, \%)\end{array}$ & $41(70.69)$ & 42(68.85) & $32(69.57)$ & $35(63.63)$ & $18(56.25)$ & $20(51.28)$ & 10(90.91) \\
\hline
\end{tabular}

\section{Trends in RR/MDR-EPTB over the 5-year period,2016-2019:}

We further analyzed the trends in RR/MDR-EPTB over a 5-year period (Figure 2a). Over the past 5 years, the proportion of RR囚MDR-EPTB cases as a

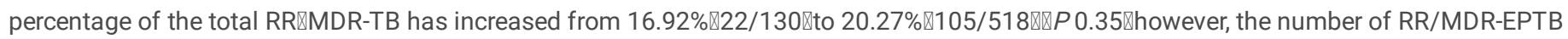
increased significantly from $22 \rrbracket 7.28 \% \varangle 22 / 302 \rrbracket$ in 2015 to $68 \rrbracket 34.77 \% \rrbracket 105 / 302 \rrbracket$ in $2019 \rrbracket P<0.01$.

As shown in Figure $2 b$, the incidence of most types of RR $₫$ MDR-EPTB except for laryngeal TB were increasing year by year during the 5 years from 2015 to 2019. Among them, lymphadenitis TB increased from 5 cases $(8.62 \%, 5 / 58)$ in 2015 to $21(36.21 \%, 21 / 58)$ cases in 2019, $P<0.01$; pleural /chest wall TB increased from 3 cases $(4.92 \%, 3 / 61)$ in 2015 to $19(31.15 \%, 19 / 61)$ cases in $2019, P<0.01$,tuberculous meningitis/CNS increased from $5 \llbracket 9.91 \%, 5 / 55 \rrbracket$ cases in 2015 to $13 \llbracket 23.64 \%, 13 / 55$ ) cases in $2019, P 0.00$; peritonitis $\llbracket$ digestive TB increased from 3 cases ( $6.52 \%, 3 / 46)$ in 2015 to $10(21.74 \%, 10 / 46)$ cases in $2019, P<0.01$; genitourinary TB increased from $1 \varangle 3.13 \%, 1 / 32 \llbracket$ case in 2015 to 9 cases $\varangle 28.12 \%, 9 / 32)$ in $2019, P$ $<0.01$; osteoarticular】spinal TB increased from 4囚10.26, 4/39凶cases in 2015 to 10 (25.64\%, 10/39) cases in $2019, P<0.01$.

\section{Drug resistance characteristics of RR/MDR-EPTB:}

The RR/MDR-EPTB was detected mainly based on Xpert $(93.71 \%, 283 / 302)$ rather than the MTB culture (56.95\%, 172/302) . Figure 3 shows the distribution of resistance to 10 anti-TB drugs of 172 culture-positive MDR-RR/EPTB strains .We found 98 (32.54 \%, 98/302) cases of pre-XDR/XDR- 
EPTB, which was significantly higher than that in MDR/RR-PTB囚18.88\%, 264/1398\during the same period. The pre-XDR/XDR-EPTB was more common in osteoarticular囚spinal TB囚61.54\%, 24/39囚and genitourinary TB (50\%, 16/32).

Resistance to ethambutol $₫ 73.51 \% \varangle 222 / 302 \rrbracket$ and streptomycin $₫ 89.07 \% \varangle 269 / 302 \rrbracket$ were common while resistance to cycloserine (4.97\%,15/302) ethionamide (3.31\% $10 / 302)$ and paminosalicylic acid $(3.31 \%, 10 / 302)$ were relatively rare. Rifabutin was sensitive to some patients $(10.26 \%$, 31/302). (Fig3)

\section{Treatment outcome:}

All 302 patients received treatment for at least 18 months. The success treatment outcome after 18 months was reported for 198 of 302 (69.56\%) MDR/RR-EPTB cases, significantly lower than that of the RR/MDR-PTB $(79.21 \%, 1105 / 1398)$ in the same period, $p<0.01$. Among them, the treatment success rate was lower in osteoarticular/spinal TB (51.28\%) and genitourinary TB (56.25\%). A total of 5 patients of MDR/RR-EPTB died during the study period as a direct result of TB. 2 were tuberculous meningitis/CNS $₫ 1$ was pleural tuberculosis complicated with tuberculous

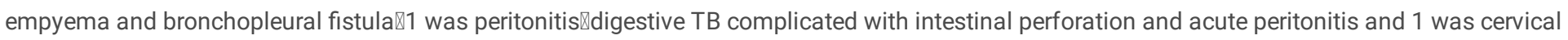
tuberculosis complicated with paraplegia.

\section{Discussion}

Datas from developed countries indicate that the incidence of EPTB is increasing as a proportion of TB (5, 8). Since EPPB is much less infectious than PTB (16), it may not have received widespread attention from governments and researchers. However, EPTB can affect almost all organs in the human body and has a wide range of clinical implications (17). Datas from a national survey show that the prevalence of smear-positive TB in China decreased from 170 cases per 100,000 population in 1990 to 59 cases per 100,000 population in 2010 (18). Despite the success of the new integrated control model, limited epidemiological information suggests that the incidence of extrapulmonary tuberculosis in China may be increasing $(3,4)$. However, all over the world including China, the in-depth epidemiological and clinical information on RR/MDR-EPTB population are still lacking. In the present study, we included 5 years of datas on all well-documented MDR-TB, in which $17.76 \%$ were EPTB and the most common site were pleura/chest wall $(20.20 \%)$ and lymphadenitis (19.21\%). Consistent with existing studies on EPTB, the majority of RR/MDR-EPTB patients were predominately female (50.66\%) $\square$ more less likely to develop diabetes (22.85\%) and more likely to be newly diagnosed ( $56.95 \%)(4,8)$. Notably, RR/ MDR-EPTB detection was more prone to delay, with a median time of $145(14,341)$ days since the first visit to hospital .This is understandable because the microbiological diagnosis of EPTB presents challenges, with having difficulty obtaining bacteriological evidence in the early stages of consultation, and some even requiring invasive biopsies or even surgery. Moreover, most of the extrapulmonary specimens were oligosaccharides at the initial stage of the disease, making the diagnosis of various tests less sensitive. For example, MTB culture reduces sensitivity to paucibacillary infection and take several weeks to produce results (19). We also found that, unlike RR/MDR-PTB, RR/MDR-EPTB relies more on Xpert to establish the diagnosis. In the study, Xpert was positive in $91.70 \%$ cases of RR/MDR-PTB and $93.71 \%$ cases of RR/MDR-EPTB, while the culture positive rate of MDR/RR-EPTB was significantly lower than that of RR/MDR-PTB. $(56.95 \%$ Vs $79.21 \%, P<0.01)$. Previous study shown that Xpert should be used as a primary diagnostic tool for the detection of EPTB, especially in tuberculous lymphadenitis. However the added value of diagnosing pleural or peritoneal tuberculosis may be limited (20) .Existing studies, however, have focused on the microbiological diagnosis of EPTB, rather than on drugresistant populations. Effective treatment of MDR-TB is based on accurate detection of drug resistance, but microbiological data and related studies on drug-resistant EPTB were still lacking. Delayed initiation of RR/MDR- EPTB treatment are likely to be associated with an increase in bacterial load and progression of the disease. Inaccurate treatment may acquire more resistance, leading to further spread of the disease (21). Rapid molecular diagnostic tests such as Xpert significantly reduce the time to diagnosis, and thus the time to treatment, compared to traditional DST (22). In our study, we found that Xpert could detect most drug-resistant EPTB and had good performance in all kinds of specimens. We believe that the main reason lies in the general lack of bacteria in conventional extrapulmonary specimens, and the development of drug-resistant MTB or infection with drug-resistant MTB may alter the nature of the oligosaccharides in the specimens. Therefore, both the culture positive rate (56.95\%) and the Xpert positive $(93.71 \%)$ rate were relative high.

Fluoroquinolones(FQs)have long been considered the cornerstone of treatment regimens for MDR-TB (23) and listed as Group A recommended drugs in the latest guidelines (15). Increased second-line drug resistance was associated with a higher rate of treatment failure (23). However, FQS has been widely used to treat bacterial infections of respiratory tract $\square$ gastrointestinal tract and urinary tract in China over the past decades (25). Improper use of FQs may lead to acquired resistance in TB patients, which in turn leads to the spread of strains that are already resistant to FQs. Molecular epidemiological studies have shown that resistance to moxifloxacin increased significantly in China between 2000 and 2010 (26). In our study, we also found $32.54 \%$ cases of pre-XDR/XDR-EPTB, which was significantly higher than that in MDR/RR-PTB $18.88 \%, P<0.01 \mathrm{Q}$, especially in osteoarticular $\square$ spinal TB $₫ 61.54 \%$ and genitourinary TB (50\%). Our results have revealed that the majority of RR / MDR-EPTB were newly diagnosed. Because the early manifestations of EPTB are indistinguishable from common bacteria infection (genitourinary infection $\square$ bone and joint infection, etc.), patients may often first referred to non-TB specialist hospitals, so antibiotics may be administered while waiting for definition. In the present study, the treatment success rate after 18 months was $69.56 \%$ in MDR/RR-EPTB, significantly lower than that of RR/MDR-PTB ( $79.21 \%$ ) in the same period. Among them, the treatment success rate was lower in osteoarticular/spinal TB (51.28\%) and genitourinary TB (56.25\%), which were also the two groups with the highest incidence of pre-XDR /XDR-EPTB. Our results also revealed that the prognosis of RR/MDR-EPTB may be worse than that of RR/MDR-PTB, and FQS resistance may be one of the main reasons. Clinicians should recommend Xpert early and use FQs cautiously in cases suspected TB. 
Our study had some limitations. EPTB was not included in routine infectious disease reports in China CDC due to its relatively low infectivity. Our retrospective study only collected datas from Shanghai pulmonary Hospital. Although the specialized hospital is the only MDR-TB designated treatment hospital in Shanghai. Our data may not be representative of the whole country. However, our findings highlight the need for rigorous management and monitoring of RR/MDR-EPTB.

\section{Conclusion}

Our study identified three main concerns for RR/MDR-EPTB. Firstly, the incidence of RR/MDR-EPTB is increasing year by year in China, which should arouse the attention of regulatory departments and non-tuberculous specialist. Secondly, the delay in the diagnosis of RR/MDR-EPTB remained long, Xpert should be recommended for all patients suspected EPTB, especially in areas where laboratory conditions for TB culture are not available. Thirdly, the level of resistance to second-line anti-TB drugs may be high ,which may lead to poor treatment outcome. These issues should be addressed in any TB surveillance and control programme implemented.

\section{Declarations}

\section{Ethics approval and consent to participate:}

The retrospective study was approved by The Ethics Committee of Shanghai Pulmonary Hospital (Approval number k17-138 ).

\section{Consent for publication:}

Not applicable

\section{Availability of data and materials:}

All datas regarding the included participants and laboratory data during the study are available from the corresponding author by email request. The study followed the CONSORT guidelines.

\section{Competing interests:}

The authors declare that they have no competing interests.

\section{Funding acknowledgements:}

This work was supported by the grant from the Shanghai Clinical Research Center for infectious disease (Tuberculosis) (19MC1910800). The funders had no role in study design, data collection and analysis, decision to publish, or preparation of the manuscript.

\section{Author contribution Statement:}

JM, WS: Data collection and sorting, statistical analysis; LF: Research design and revised the manuscript. SW,CL,TL: Manuscript writing. All authors read and approved the final submitted version.

\section{Acknowledgements:}

We thank all participants for their time and efforts.

\section{References}

1. WHO. Global tuberculosis report 2020. Geneva: World Health Organization; 2020.

2. Solovic I, Jonsson J, Korzeniewska-Koseła M, Chiotan DI, Pace-Asciak A, Slump E, et al. Challenges in diagnosing extrapulmonary tuberculosis in the European Union, 2011. Euro Surveill. 2013;18:20432

3. Wang X, Yang Z, Fu Y, Zhang G, Wang X, Zhang Y, et al. Insight to the epidemiology and risk factors of extrapulmonary tuberculosis in Tianjin, China during 2006-2011. PLoS One. 2014;9:e112213.

4. Pang Yu, An Jun, Shu Wei et al. Epidemiology of Extrapulmonary Tuberculosis among Inpatients, China, 2008-2017 .Emerging Infect. Dis., 2019, 25: 457-464.

5. Sandgren A, Hollo V, van der Werf MJ. Extrapulmonary tuberculosis in the European Union and European Economic Area, 2002 to 2011. Euro Surveill. 2013;18:20431.

6. Kohli M, Schiller I, Dendukuri N, Yao M, Dheda K, Denkinger CM, Schumacher SG, Steingart KR. Xpert MTB/RIF Ultra and Xpert MTB/RIF assays for extrapulmonary tuberculosis and rifampicin resistance in adults. Cochrane Database of Systematic Reviews 2021 , Issue 1.

7. Hayward S E, Rustage K, Nellums L B, et al. Extrapulmonary tuberculosis among migrants in Europe, 1995 to 2017 - ScienceDirect [J]. Clinical Microbiology and Infection, 2020. 
8. Peto HM, Pratt RH, Harrington TA, LoBue PA, Armstrong LR. Epidemiology of extrapulmonary tuberculosis in the United States, 1993-2006. Clin Infect Dis. 2009;49:1350-7

9. World Health Organization (WHO). Extensively DR tuberculosis (XDR.TB): recommendations for prevention and control. Wkly Epidemiol Rec. 2006;81:430-2.

10. Telenti A, Imboden P, Marchesi F, Lowrie D, Cole S, Colston MJ, et al.Detection of rifampicin-resistance mutations in Mycobacterium tuberculosis . Lancet 1993;341(8846):647-50.

11. World Health Organization. Rapid implementation of the Xpert MTB/RIF diagnostic test. Technical and operational 'How-to'. Practical considerations. who.int/tb/ publications / tb- amplificationtechnology-implementation/en/ 2011

12. World Health Organization. Global tuberculosis report 2018. Geneva, Switzerland: World Health Organization, 2018.

13. GLI model TB diagnostic algorithms. Global Laboratory Initiative; 2017. http:// www. stoptb. org/ wg/gli/ assets/ documents / GLI_ algorithms.pdf.

14. Wu Zheyuan, Rueda Zulma Vanessa,Li Tao et al. Effect of the Xpert MTB/RIF on the detection of pulmonary tuberculosis cases and rifampicin resistance in Shanghai, China.[J] .BMC Infect Dis, 2020, 20: 153.

15. WHO. WHO consolidated guidelines on drug-resistant tuberculosis treatment. world health organization. $2019 ; 2019$.

16. Gomes T, Reis-Santos B, Bertolde A, Johnson JL, Riley LW, Maciel EL. Epidemiology of extrapulmonary tuberculosis in Brazil: a hierarchical model. BMC Infect Dis. 2014;14:9.

17. Kruijshaar ME, Abubakar I. Increase in extrapulmonary tuberculosis in England and Wales 1999-2006. Thorax. 2009;64:1090-5.

18. Wang L, Zhang H, Ruan Y, Chin DP, Xia Y, Cheng S, et al. Tuberculosis prevalence in China, 1990-2010; a longitudinal analysis of national survey data. Lancet. 2014;383:2057-64

19. Kohli M, Schiller I, Dendukuri N, Yao M, Dheda K, Denkinger CM, Schumacher SG, Steingart KR. Xpert MTB/RIF Ultra and Xpert MTB/RIF assays for extrapulmonary tuberculosis and rifampicin resistance in adults. Cochrane Database of Systematic Reviews 2021 , Issue 1.

20. Tadesse M,Abebe G,Bekele A et al. Xpert MTB/RIF assay for the diagnosis of extrapulmonary tuberculosis: a diagnostic evaluation study .Clin Microbiol Infect, 2019, 25: 1000- 1005.

21. Yang C, Luo T, Shen X, Wu J, Gan M, Xu P, Wu Z, Lin S, Tian J, Liu Q, Yuan Z, Mei J, DeRiemer K, Gao Q. 2017. Transmission of multidrugresistant Mycobacterium tuberculosis in Shanghai, China: a retrospective observational study using whole-genome sequencing and epidemiological investigation. Lancet Infect Dis 17:275-284.

22. Boehme CC, Nicol MP, Nabeta P, Michael JS, Gotuzzo E, Tahirli R, Gler MT, Blakemore R, Worodria W, Gray C, Huang L, Caceres T, Mehdiyev R, Raymond L, Whitelaw A, Sagadevan K, Alexander H, Albert H, Cobelens F, Cox H, Alland D, Perkins MD. 2011. Feasibility, diagnostic accuracy, and effectiveness of decentralised use of the Xpert MTB/RIF test for diagnosis of tuberculosis and multidrug resistance: a multicentre implementation study. Lancet 377:1495-1505.

23. Collaborative Group for the Meta-Analysis of Individual Patient Data in MDR-TB treatment-2017,Ahmad Nafees,Ahuja Shama D et al. Treatment correlates of successful outcomes in pulmonary multidrug-resistant tuberculosis: an individual patient data meta-analysis.Lancet, 2018, 392: 821-834.

24. D. Falzon, N. Gandhi, G.B. Migliori, G. Sotgiu, H.S. Cox, T.H. Holtz, et al. Resistance to fluoroquinolones and second-line injectable drugs: impact on multidrug-resistant TB outcomes. Eur Respir J, 42 (1) (2012), pp. 156-168

25. Z. Zhang, J. Lu, Y. Wang, Y. Pang, Y. Zhao.Prevalence and molecular characterization of fluoroquinolone-resistant Mycobacterium tuberculosis isolates in China.Antimicrob Agents Chemother, 58 (1) (2014), pp. 364-369

26. Y. Pang, J. Lu, F. Huo, Y. Ma, L. Zhao, Y. Li, et al. Prevalence and treatment outcome of extensively drug-resistant tuberculosis plus additional drug resistance from the national clinical center for tuberculosis in china: a five-year review. J Infect (2017), Article S0163445317302621

\section{Figures}




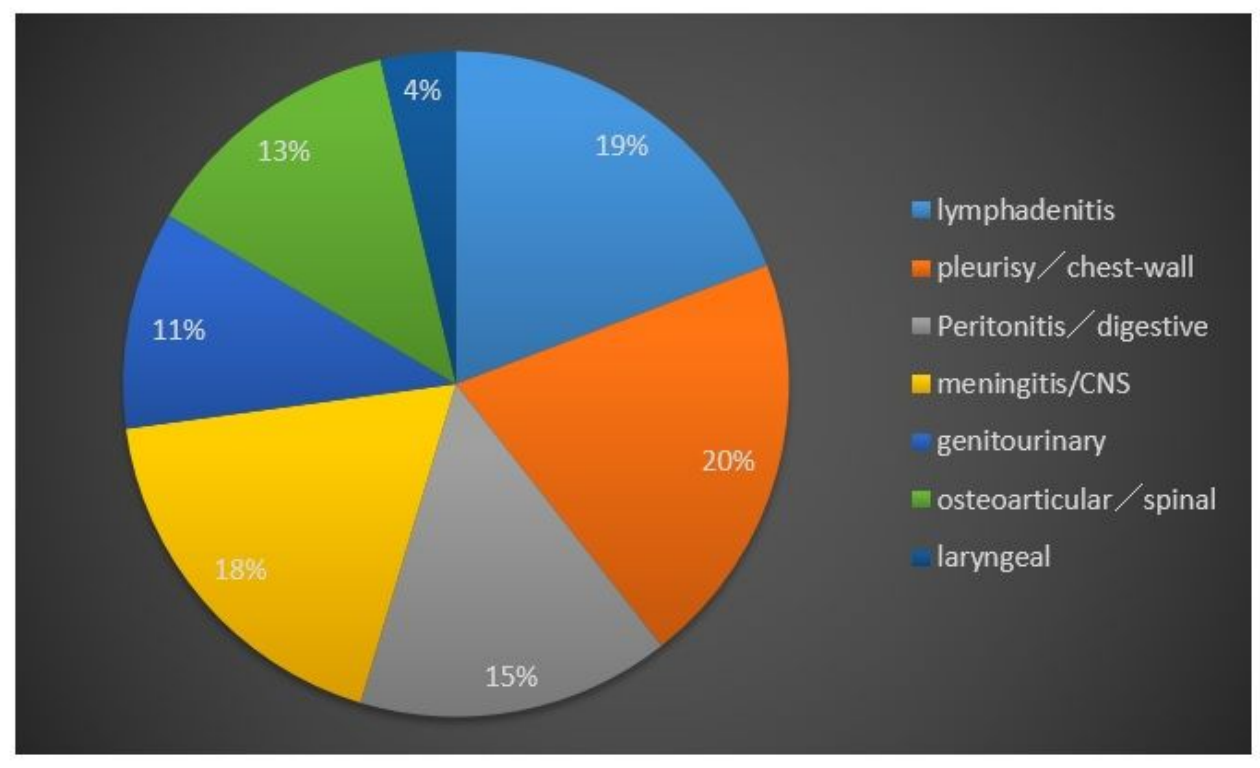

\section{Figure 1}

The anatomical site of RR/MDR-EPTB

\section{Fig $2 a$}

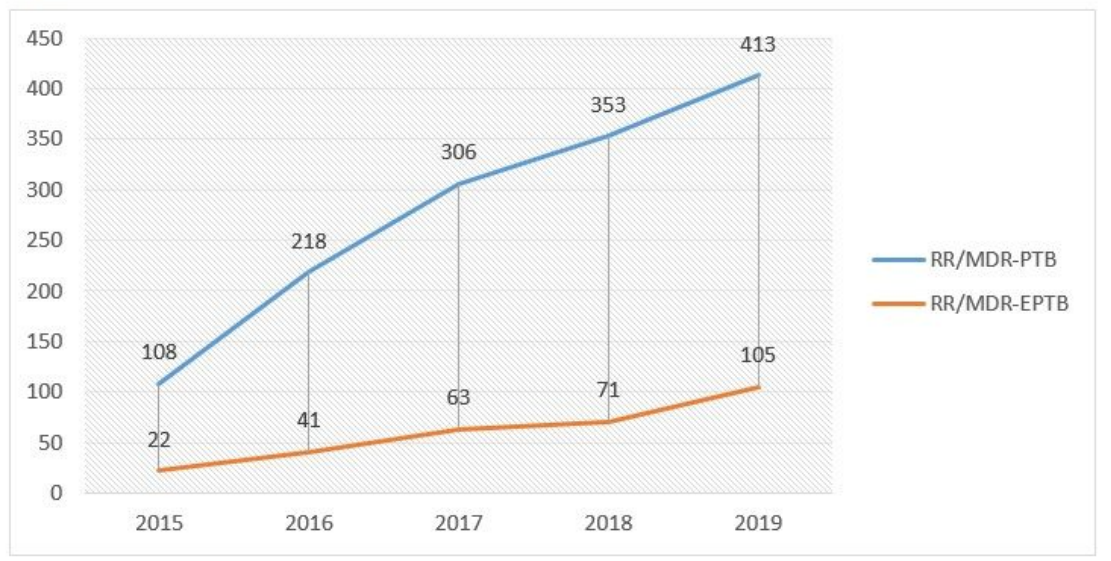

Fig $2 b$

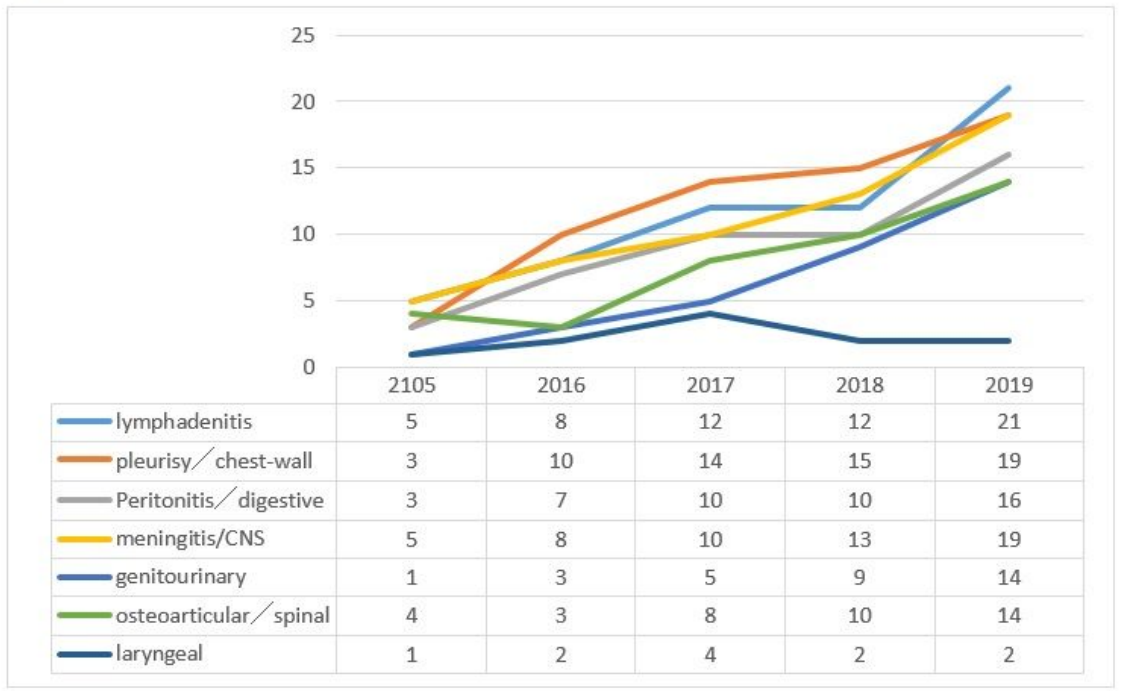

Figure 2 
2a Relative rates of RR/MDR EPTB and RR/MDR-PTB in the same Period, 2015-2019 (n) 2b Annual prevalence and site of RR/MDR-EPTB, 2015-2019 (n)

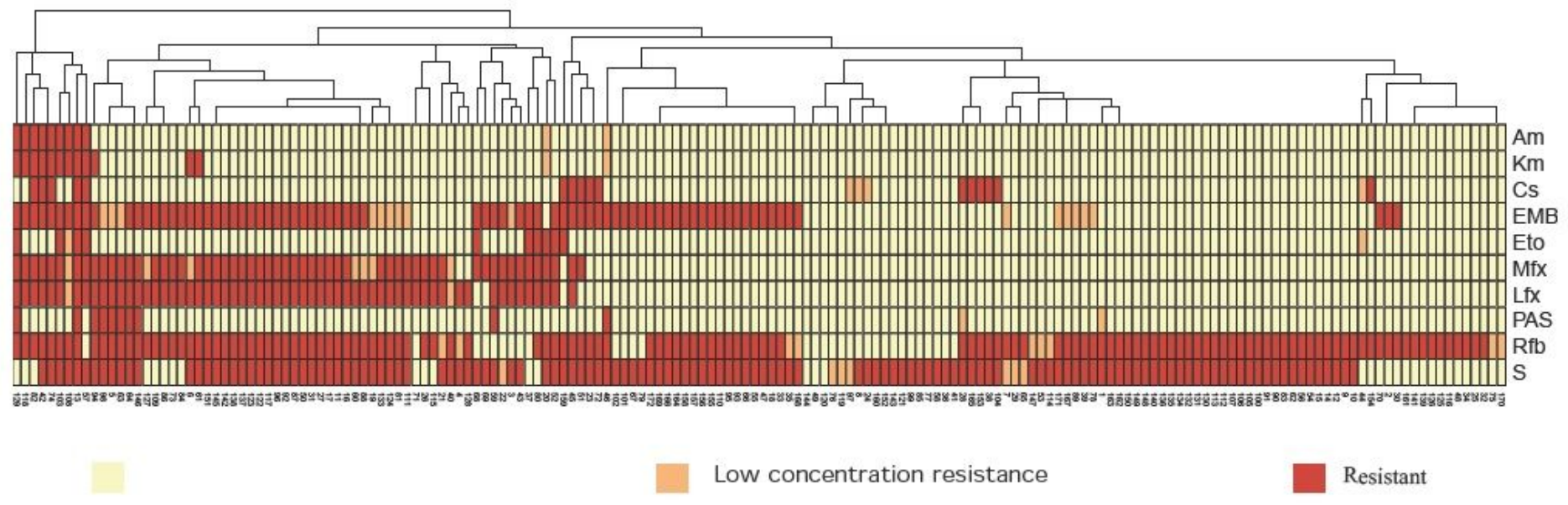

\section{Figure 3}

Figure shows the distribution of resistance to 10 anti-TB drugs of 172 culture-positive MDR-RR/EPTB strains 